\title{
Quantum Computing Based Technique for Cancer Disease Detection System
}

\author{
Milan Jain* and Setu Kumar Chaturvedi
}

Department of Computer Science and Engineering, Technocrats Institute of Technology, Bhopal, India

\begin{abstract}
In present scenario where various diseases are here and out of them the cancer is one of them most devastating disease. Presently the detection procedure is very much time consuming and the results obtain by them are not so fast, so here in this respect there is a need of more accurate, fast and efficient method through computing technologies. This research work incorporates Quantum computing with the clustering algorithm i.e. Shor Algorithm of Quantum Computing with various Hierarchical Clustering Technique. The Hierarchical Clustering Technique helps in clustering of results to obtain an approach for Cancer Disease Detection. Now accurate results are obtained, when adopting Shor Algorithm of quantum computing in disease detection technique with different Hierarchical Clustering. Here the Shor Algorithm helps to increase the efficiency in term of accuracy and Hierarchical Clustering Technique helps to detect the stages of Cancer. So, the proposed system gives a new approach in the field of cancer disease diagnosis. The results obtained by different Hierarchical Clustering Techniques are discussed.
\end{abstract}

Keywords: Quantum computing; Cancer detection; Hierarchical clustering technique; Quantum shear sorting

\section{Introduction}

One of the fastest growing areas of research and technical field is Quantum computing, which revolves two area of century, computer science (including information science), and quantum mechanics. A simple computer operate any operation at a time due to this it takes too time for solve the problem and this is overcome by quantum computer [1].

In classical computing, information unit is a bit. The physical states of bits are two logically known as bit 1 and bit 0 . These are commonly realized by physical ON state and OFF state of transistor. In quantum computing, equivalently two quantum states define basic information processing unit known as qubit. The representation of qubit 0 and 1.In 1982, the Nobel prize-winning physicist Richard Feynman thought up the idea of a 'quantum computers', a device which uses the effects of quantum mechanics to its advantage [2].

In the classical model of computing, the most common building block is bit, it can only lie in two distinct states, 0 or 1 . In quantum computers the rules are changed [2-4]. Not only can a 'quantum bit', usually referred to as a 'qubit', exist in the classical 0 and 1 states, this also be in a coherent superposition of both states.

This is just an insight into some of the startling application of Quantum Computing to illustrate their immense power and capability. Feynman states that quantum computers would be able to simulate quantum mechanics systems with a highly greater degree of accuracy than is possible with classical computers [5].

\section{Fundamentals of cancer disease}

Term Cancer denotes malignant neoplasm, characterized as class of diseases involving uncontrolled cell growth. This $\mathrm{c}$ an harm the human body due to spread (movement) of damage cells in different parts of body with the help of blood stream. Human beings are affected by about 200 different types of known cancers [6].

The $\mathrm{c}$ ancer i s $\mathrm{n}$ ot e asy $\mathrm{t}$ o d efine as a re sult the ce lls gr ow uncontrollable. The cancer causes are diverse, various, tough, and only half understood till date. There are many reasons which are responsible for cancer like use of tobacco, alcohol, user diet, some infections, radiation, poor physical activity, lifestyle and environmental pollutants
[7]. These source can injured or combine genes along with present genes due to which cells undergoes cancerous mutations [8]. Near about $5-10 \%$ cancers find directly for inherited genetic defects [9]. The detection of Cancer disease can be done by numerous ways like presence of some signs and symptoms, screen testing, microscopic examination of a collected tissue sample. There are many possibilities for survival of this disease by its type and the location where the cancer is situated.

\section{Literature Survey}

This section reviews the current literature and related work in the areas of cancer disease prediction with different methods and technology is given below:

Quantum algorithms that outperform classical computation have focused primarily on the non Abelian hidden subgroup problem, which generalizes the central problem solved by Shor's factoring algorithm. We suggest an alternative generalization, namely to problems of finding hidden nonlinear structures over finite fields. We give examples of two such problems that can be solved efficiently by a quantum computer, but not by a classical computer. We also give some positive results on the quantum query complexity of finding hidden nonlinear structures [10].

A modern web-based management system that allows integrating different sources, validating and elaborating data thus providing a new evaluation system for the oncology network based on cancer registries. We developed a Web 2.0 management system for the Umbria Cancer Registry based on AMPAX technology. It is modular and extensible and information consistency is guaranteed by entity relationship principles.

${ }^{*}$ Corresponding author: Milan Jain, Department of Computer Science and Engineering, Technocrats Institute of Technology, Bhopal, India, Tel: 9907027711; E-mail: jain.milan.jain@gmail.com

Received March 18, 2014; Accepted April 20, 2014; Published April 23, 2014

Citation: Jain M, Chaturvedi SK (2014) Quantum Computing Based Technique for Cancer Disease Detection System. J Comput Sci Syst Biol 7: 095-102. doi:10.4172/jcsb.1000143

Copyright: (c) 2014 Jain M, et al. This is an open-access article distributed under the terms of the Creative Commons Attribution License, which permits unrestricted use, distribution, and reproduction in any medium, provided the original author and source are credited. 
Cancer sites, topology, morphology, and behaviour are coded according to the International Classification of Diseases. S.G.RTUP is integrated with the Oncology Network and gives timely epidemiological indices for evaluation of ontological activities. Automatic importing of routinely available data from pathology archives, screening services, and hospital discharge records will reduce the time needed to produce data and will also allow the expansion of registered information. Several services for data visualization and statistical analysis are implemented [11].

Computing is perhaps one of the most distinguished features that differentiate humans from animals. Aside from counting numbers using fingers and toes, abacus was the first great computing machine of human civilization. Over thousands of years, the development of mechanical computing machines had been slow. Quantum computers are a new type of computing machine which works on the principles of the microscopic world, quantum mechanics. There have been intensive worldwide efforts in physically building such machines. To build such a machine, the system must be able to provide a sequence of two-level quantum sub-systems that serve as quantum bits, or qubits for short. Secondly, the quantum system should be initializable to some desired states. Thirdly, it should be robust against environmental disturbance which is translated into a long coherence time in the terminology of physics. Fourthly, the system should accommodate at least a set of basic universal gates so that any unitary operations performed in a quantum computer can be constructed from them. Finally, we should be able to measure the quantum system to read out the results of the computation [12].

The authors propose a regulation-level representation for microarray data and optimize it using genetic algorithms (GAs) for cancer classification. Compared with the traditional expression-level features, this representation can greatly reduce the dimensionality of microarray data and accommodate noise and variability such that many statistical machine-learning methods now become applicable and efficient for cancer classification. Experimental results on real-world microarray datasets show that the regulation-level representation can consistently converge at a solution with three regulation levels [13].

There has been many skewed cancer gene expression datasets in the post-genomic era. Extraction of differential expression genes or construction of decision rules using these skewed datasets by traditional algorithms will seriously underestimate the performance of the minority class, leading to inaccurate diagnosis in clinical trials. It presents a skewed gene selection algorithm that introduces a weighted metric into the gene selection procedure. The extracted genes are paired as decision rules to distinguish both classes, with these decision rules then integrated into an ensemble learning framework by majority voting to recognize test examples; thus avoiding tedious data normalization and classifier datasets [14].

An accurate and reliable decision making in ontological prognosis can help in the planning of suitable surgery and therapy, and generally, improve patient management through the different stages of the disease. The aim is to investigate the fuzzy-nearest neighbour (FK-NN) classifier as a fuzzy logic method that provides a certainty degree for prognostic decision and assessment of the markers, and to compare it with [15].

In quantum circuits, importing of additional qubits can reduce the operation time and prevent decoherence induced by the environment. The author described how to relax existing qubits without additional qubits to significantly reduce the operation time of the quantum Fourier circuit compared to a circuit without optimization. The results indicate that this scheme makes full use of the qubits relaxation. The concepts can be applied to improve similar quantum circuits and guide the physical implementations of quantum algorithms or devices [16].

Quantum switching architecture for nearest neighbour coupling. An efficient quantum shear sorting (QSS) algorithm is used to reduce the number of time steps. For the QSS algorithm, the running complexity of the quantum switching architecture is polynomial in time with the nearest neighbour coupling and the implementation is less complex. The result shows that improved switching is extremely simple to implement using existing quantum computer candidates [17].

The author demonstrated that clinicians exhibit both interexpert and intra-expert variability when making difficult decisions. In contrast, the vast majority of computerized models that aim to provide automated support for such decisions do not explicitly recognize or replicate this variability. The perfect consistency of computerized models is often presented as a de facto benefit. The author describes a novel approach to incorporate variability within a fuzzy inference system using non-stationary fuzzy sets in order to replicate human variability [18].

Hybrid multi-layered Group Method of Data Handling (GMDH)type neural network algorithm using principal component-regression analysis is proposed and applied to the computer aided image diagnosis (CAD) of lung cancer. In the GMDH-type neural network, heuristic self-organization method, which is a kind of evolutional computation, is used to organize the neural network architecture. But, multi-co linearity occurs and prediction values become unstable. Hybrid multilayered GMDH-type neural network using principal componentregression analysis is proposed [19].

Terahertz reflection imaging (at frequencies_0.1-10 THz/1012 $\mathrm{Hz}$ ) is non-ionizing and has potential as a medical imaging technique; however, there is currently no consensus on the optimum imaging parameters to use and the procedure for data analysis. This may be holding back the progress of the technique. This article describes the use of various intelligent analysis methods to choose relevant imaging parameters and optimize the processing of terahertz data in the diagnosis of ex vivo colon cancer samples [20].

The aim at finding the smallest set of genes that can ensure highly accurate classification of cancers from microarray data by using supervised machine learning algorithms [21].

The author gives a simple proof of why a quantum computer, despite being in all possible states simultaneously, needs at least $0.707 \sqrt{ } \mathrm{N}$ queries to retrieve a desired item from an unsorted list of $\mathrm{N}$ items. The proof is refined to show that a quantum computer would need at least $0.785 \sqrt{ } \mathrm{N}$ queries. The quantum search algorithm needs precisely this many queries [22].

The quantum search algorithm is a technique for searching possibilities in only steps. Although the algorithm itself is widely known, not so well known is the series of steps that first led to it, these are quite different from any of the generally known forms of the algorithm. The author describes these steps, which start by discretizing Schrödinger's equation and also provides a self-contained introduction to quantum computing algorithms from a new perspective [23].

Probabilistic neural networks (PISMs) classification of cancer cell image is described. This network is simpler and faster than backpropagating neural networks (BPNNs) during training and learning. Neural networks combined with expert experience is presented in 
order to improve the classification accuracy of the networks and the simulation experiments were performed and the results have shown that the method presented is very efficient and feasible [24].

Breast cancer screening has reference to screening of asymptomatic, generally healthy women for breast cancer, to identify those who should receive a follow up check. Early screening can detect noninvasive ductile carcinoma in situ (called "pre breast cancer"), which almost never forms a lump and is generally non-detectible, except by mammography. This will describe the design and preliminary evaluation of this PNN/GRNN ensemble pre-screener, in the context of a possible pre-screening protocol, which may, if required, include other data. The results show that using the ensemble technique provides almost a $20 \%$ AUC increase over the average standalone PNN and almost $10 \%$ over the best performing PNN [25].

As in classical coding theory, quantum analogs of low-density parity-check (LDPC) codes have offered good error correction performance and low decoding complexity by employing the Calder bank Shor Steane construction. However, special requirements in the quantum setting severely limit the structures such quantum codes can have. While the entanglement-assisted stabilizer formalism overcomes this limitation by exploiting maximally entangled states (ebits), excessive reliance on ebits is a substantial obstacle to implementation. The author provides necessary and sufficient conditions for the existence of quantum LDPC codes which are obtainable from pairs of identical LDPC codes and consume only one ebit, and studies the spectrum of attainable code parameters [26].

\section{Proposed System}

The Quantum Computing technique can provide faster and efficient results with the use of different parameter in this system and the K-means clustering technique and Agglomerative clustering technique can provide a result analysis by clustering of results. For this $1^{\text {st }}$ collect the information in the form of tumor size and node status as Dataset, which has to be analyzed by both conventional pathological tests \& our Quantum computing based technique which involves Shor Algorithm as a analyzing tool to analyze the dataset.

In the next step the clustering of obtained result by both techniques is done. In case of analyses of dataset with conventional pathological tests these analyze different aspects one by one in stages i.e. after completion of $1^{\text {st }}$ they proceed towards $2^{\text {nd }}$ and so on, whereas on the other hand in our Quantum computing based Analyses Shor Algorithm analyze the dataset peculiarly on the basis of range of parameters so there is no such time consumption, here if the parameters is not in the range of define values so it automatically analyze on next level and gave results accordingly and then treatment will start within a short duration with more accuracy according to cancer type and stage of severity, because after getting results from Shor Algorithm and clustering an oncologist can get a clear cut idea about the stage of Cancer in which patient held (Figure 1).

\section{Proposed algorithm}

Basically six steps are used for making to create a new dataset which are given below:

Step 1: Apply the Shor Algorithm on dataset providing tumor size denoted by 'A', Metastasis denoted by 'MS' and Node status 'NS'.

Step 2: Apply the number ' $\mathrm{N}$ ' is the number wish to factorize. As the different stages of Cancer depending upon severity are here and the remainder from this operation is placed in a second 3 bit register.

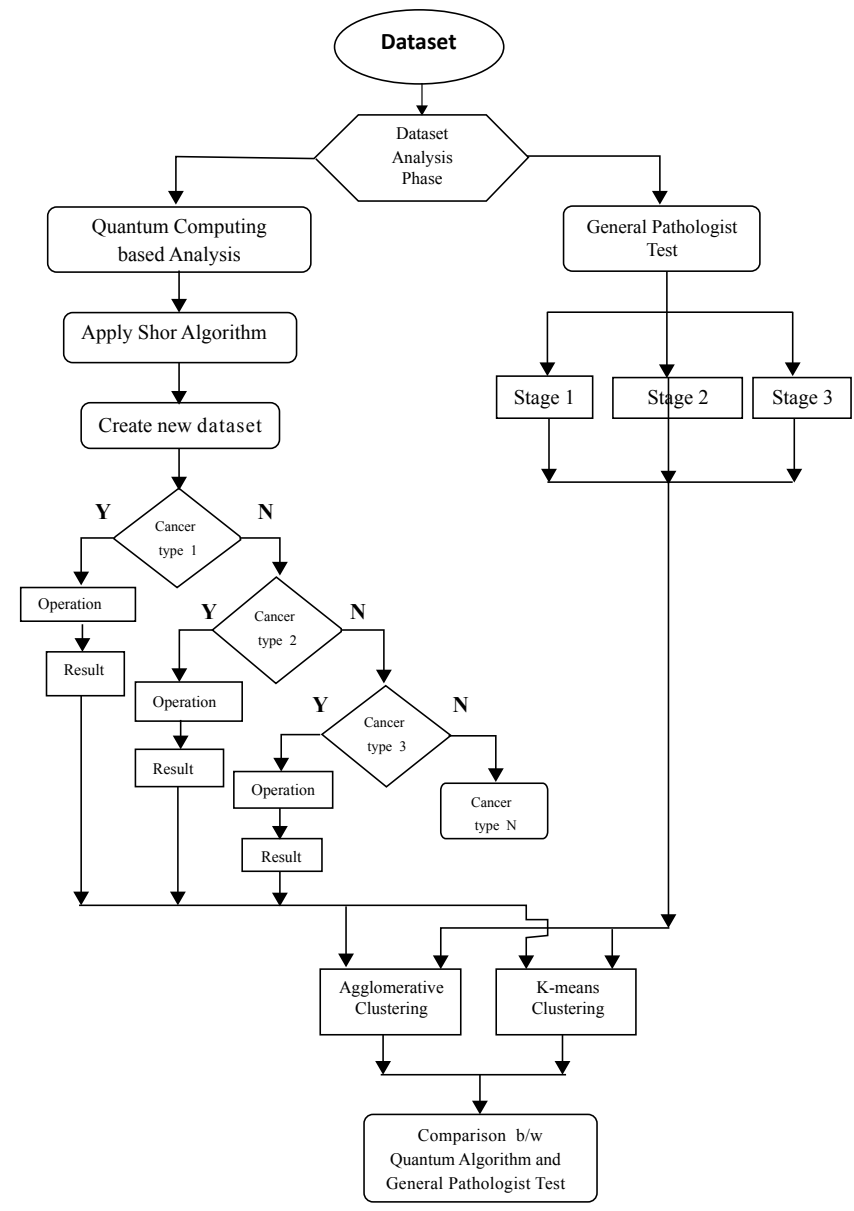

Figure 1: Designed System for Cancer Disease Detection.

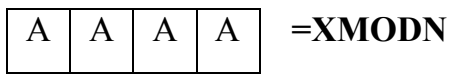

Step 3: Apply random number $\mathrm{X}$, where $1<\mathrm{X}<\mathrm{N}-1$. This is the last stage of providing the initials to execute the Algorithm and there are chances of multiple answers so to reduce the error in operation these to be verified with different value of random number ' $\mathrm{X}$ '.

Step 4: Results provided from Shor Algorithm. The result which we get is in the form of stages of Cancer which are now ready for clustering by using statistical techniques.

Step 5: Clustering by K-means Clustering Technique and Agglomerative clustering technique, here top to bottom approach used. Clustered values are used to draw Dendrogram and graphical representations.

Step 6: Comparing the clustered values of results obtained by both the techniques i.e. from conventional Cancer Disease Detection and Shor Algorithm based Disease Detection technique.

\section{Experimental Setup}

For clustering of result here the use of Hierarchical Clustering Explorer is found logical. HCE is visualization for interactive exploration of $\mathrm{m}$-dimension data.

It is to help users explore and taken knowledge of multidimensional 
Citation: Jain M, Chaturvedi SK (2014) Quantum Computing Based Technique for Cancer Disease Detection System. J Comput Sci Syst Biol 7: 095102. doi: $10.4172 / j c s b .1000143$

datasets by maximize the human understanding skills that have been underutilized. By the help of this we can systematically scrutinize multidimensional datasets in a way to identify unexpected interesting features hidden in the multidimensional space.

\section{Linkage method}

When hierarchical clustering algorithm merges two clusters to generate a new bigger cluster, it should calculate the distances of the previous and next clusters. We have 5 different linkage methods. Let $\mathrm{C}_{n}$ be a new cluster, a merge of $C_{i}$ and $C_{i \cdot}$. Let $C_{k}$ be a left. Average Linkage (UPGMA: Unweighted Pair Group Method with Arithmetic Mean)

$$
\operatorname{DIST}\left(C_{n}, C_{k}\right)=\frac{\left|C_{i}\right|}{\left|C_{i}\right|+\left|C_{j}\right|} \operatorname{DIST}\left(C_{j}, C_{k}\right)+\frac{\left|C_{j}\right|}{\left|C_{i}\right|+\left|C_{j}\right|} \operatorname{DIST}\left(C_{j}, C_{k}\right)
$$

- Average Group Linkage (Centroid Linkage) :

$$
\operatorname{DIST}\left(C_{n}, C_{k}\right)=\operatorname{DIST}\left(\operatorname{Mean}\left(C_{n}\right), \operatorname{Mean}\left(C_{k}\right)\right)
$$

- Complete Linkage :

$$
\operatorname{DIST}\left(C_{n}, C_{k}\right)=\operatorname{Max}\left(\operatorname{DIST}\left(C_{j}, C_{k}\right), \operatorname{DIST}\left(C_{j}, C_{k}\right)\right)
$$

- $\quad$ Single Linkage :

$$
\operatorname{DIST}\left(C_{n}, C_{k}\right)=\operatorname{Min}\left(\operatorname{DIST}\left(C_{j}, C_{k}\right), \operatorname{DIST}\left(C_{j}, C_{k}\right)\right)
$$

- Shneiderman's 1 by 1 Linkage :

Let $\mathrm{C}_{n-1}$ be the newly merged cluster in the previous iteration. Let $\mathrm{C}_{m}$ be the closest cluster to $\mathrm{C}_{n-1}$, and $\mathrm{C}_{p}$ be the closest cluster to $\mathrm{C}_{m}$.

If $\mid$ DIST $\left(\mathrm{C}_{n-1}, \mathrm{C}_{m}\right)-\operatorname{DIST}\left(\mathrm{C}_{m}, \mathrm{C}_{p}\right) \mid<$ THRESHOLD, merge $\mathrm{C}_{n-1}$ and $\mathrm{C}_{m}$ instead of searching two new closest clusters globally.

\section{Experiment results and analysis}

Experiments performed on dataset of cancer: In this Dataset which have collected data for Cancer Disease which contain the Tumor Size, Node Status, Metastasis (Collected from Navodaya Cancer Hospital, Indrapuri, Bhopal). These three parameters have a great importance in Cancer Disease detection as Tumor size in any type of Cancer is the first Criteria for further analyses then Metastasis i.e. the spread of a cancer from one organ or part to another non-adjacent organ or part and Node status i.e. the lymph node condition at the site of tumours which either be negative or positive (Table 1).

Experiments performed on proposed dataset of cancer: The given below variables are used for applying the Shor Algorithm on dataset (Table 2)

Here $\mathrm{A}$ is the tumor size,

$\mathrm{X}$ is the random number,

$\mathrm{N}$ is the number we wish to factorized

M denote the metastasis

$\mathrm{M} 0=$ no spread of tumor

M1=tumor has spread

NS denote the node status

\begin{tabular}{|l|l|l|l|l|l|l|l|}
\hline S.no & Name & ID & A & NS & MS & Age & Weight \\
\hline 1 & Ankya & 1 & 2 & N0 & M0 & 18 & 45 \\
\hline 2 & Sujit & 5 & 2 & N0 or N1 & M0 & 20 & 78 \\
\hline 3 & Dinesh & 6 & 3 & N0 or N1 & M0 & 24 & 68 \\
\hline 4 & Ruchi & 9 & 7 & N1 & M1 & 28 & 55 \\
\hline 5 & Kiran & 11 & 1 & N0 & M0 & 49 & 62 \\
\hline 6 & Dimple & 15 & 3 & N0 or N1 & M0 & 51 & 56 \\
\hline 7 & Rohan & 21 & 4 & N0 or N1 & M1 & 44 & 72 \\
\hline 8 & Pradeep & 26 & 4 & N0 or N1 & M0 & 52 & 69 \\
\hline 9 & Servesh & 31 & 1 & N0 & M0 & 40 & 73 \\
\hline 10 & Vinay & 35 & 6 & N0 & M0 & 53 & 75 \\
\hline 11 & Ashish & 39 & 4 & N0 or N1 & M1 & 67 & 70 \\
\hline 12 & Shanti & 41 & 1 & N0 & M0 & 30 & 64 \\
\hline 13 & Upendra & 45 & 3 & N0 or N1 & M0 & 26 & 67 \\
\hline 14 & Kapil & 2 & 3 & N0 or N1 & M0 & 44 & 70 \\
\hline 15 & Sanjeev & 12 & 4 & N0 & M0 & 73 & 55 \\
\hline 16 & Sanchita & 14 & 2 & N0 or N1 & M1 & 81 & 52 \\
\hline 17 & Shobhit & 24 & 3 & N0 or N1 & M0 & 54 & 48 \\
\hline 18 & Nirupma & 34 & 2 & N0 or N1 & M0 & 31 & 53 \\
\hline
\end{tabular}

Table 1: Dataset of Cancer Disease Here, $A$ is the Tumor size

$$
\begin{aligned}
& \text { ID is Identification number } \\
& \text { N0 is clear or Negative node } \\
& \text { N1 is Cancerous or positive node } \\
& \text { M0 is No spread of Tumor } \\
& \text { M1is Tumor has spread } \\
& \text { Weight in Kg and Age in years }
\end{aligned}
$$

\begin{tabular}{|l|l|l|l|l|l|l|l|l|}
\hline S.no & Name & ID & A & X & N & STAGE & NS & MS \\
\hline 1 & Ankya & 1 & 2 & 2 & 3 & 1 & N0 & M0 \\
\hline 2 & Sujit & 5 & 2 & 4 & 7 & 2 & N0 or N1 & M0 \\
\hline 3 & Dinesh & 6 & 3 & 2 & 5 & 3 & N0 or N1 & M0 \\
\hline 4 & Ruchi & 9 & 7 & 6 & 7 & 4 & N1 & M1 \\
\hline 5 & Kiran & 11 & 1 & 2 & 3 & 0 & N0 & M0 \\
\hline 6 & Dimple & 15 & 3 & 3 & 5 & 2 & N0 or N1 & M0 \\
\hline 7 & Rohan & 21 & 4 & 3 & 7 & 4 & N0 or N1 & M1 \\
\hline 8 & Pradeep & 26 & 4 & 3 & 6 & 3 & N0 or N1 & M0 \\
\hline 9 & Servesh & 31 & 1 & 1 & 3 & 0 & N0 & M0 \\
\hline 10 & Vinay & 35 & 6 & 4 & 7 & 1 & N0 & M0 \\
\hline 11 & Ashish & 39 & 4 & 2 & 6 & 4 & N0 or N1 & M1 \\
\hline 12 & Shanti & 41 & 1 & 3 & 4 & 0 & N0 & M0 \\
\hline 13 & Upendra & 45 & 3 & 2 & 5 & 3 & N0 or N1 & M0 \\
\hline 14 & Kapil & 2 & 3 & 3 & 5 & 2 & N0 or N1 & M0 \\
\hline 15 & Sanjeev & 12 & 4 & 2 & 5 & 1 & N0 & M0 \\
\hline 16 & Sanchita & 14 & 2 & 4 & 6 & 4 & N0 or N1 & M1 \\
\hline 17 & Shobhit & 24 & 3 & 2 & 5 & 3 & N0 or N1 & M0 \\
\hline 18 & Nirupma & 34 & 2 & 3 & 7 & 2 & N0 or N1 & M0 \\
\hline
\end{tabular}

Table 2: Results obtained from Shor Algorithm based Cancer Disease Detection Technique.

\section{$\mathrm{N} 0=$ clear or negative nodes}

$\mathrm{N} 1=$ cancerous or positive node

Shor based algorithm shows the stages of cancer, Patient age in years and ID shows the identification of patients. First evaluate the dataset on the basis of Tumor Size then after applying Shor Algorithm get different Stages according to Tumor Size and Node status and Metastasis condition. According to Node Status and Metastasis Conditions sometime a patient who having a large Tumor may also do not have any Cancer Disease because of having N0 which means negative node or M0 condition which means Tumor is not malignant or not spreading which shows the Tumor is here but not carcinogenic. 


\section{Results}

Results comparison between conventional cancer disease detection technique and shor algorithm based cancer disease detection technique on the basis of dendrogram

For comparison of results we divide this in two sections the dendrogram of conventional cancer disease detection technique contains the Dendrogram of Conventional Technique whereas Dendrogram of Shor algorithm based cancer disease detection technique contain the Dendrogram of Shor Algorithm based Technique. Here these are used as with the help of different color intensities elaboration of results is more convinent.

Dendrogram of conventional cancer disease detection technique: In this section the Results for Conventional Cancer Disease Detection System in the form of Dendrogram are illustrated (Figure 2).

In Figure 2 tumor size, Stages of Cancer and Name of patient are shown. The main purpose of Dendrogram display is to determine the natural clusters with the help of different color clusters. Here in this Dendrogram the uppermost green layer denotes the Tumor size (A), middle layer shows Stages of Cancer and lower one is for Id wise clusters. With the advancement in stages of Cancer and Size of Tumor the color intensity gradually increases from light green to dark then in extreme cases i.e. Tumor size above 5 and for stages above 4 it changes to red. Here in Conventional Cancer disease detection Technique only Tumor Size is the parameter which is use in most of the cases so, in complex cases where lymph node status is not carcinogenic but according to tumor size this technique shows more advance stages which may not be true for each instance.

Dendrogram of Shor algorithm based cancer disease detection technique

For Agglomerative hierarchical clustering technique: The Result of Agglomerative Hierarchical clustering technique for Shor Algorithm based Cancer disease detection System these Dendrogram are shows

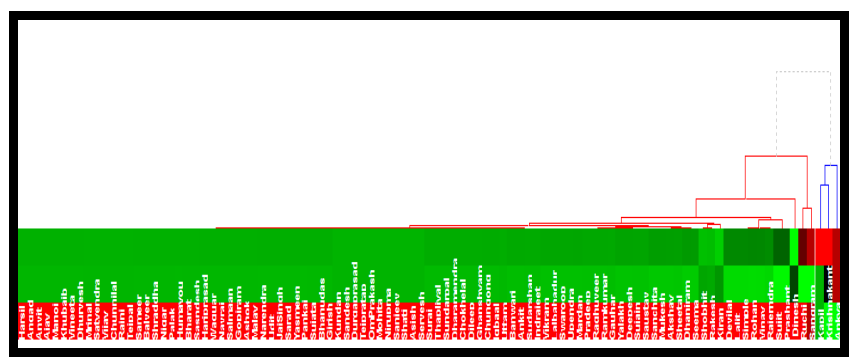

Figure 2: Dendrogram of Conventional Cancer disease detection Technique using Euclidean Distance.

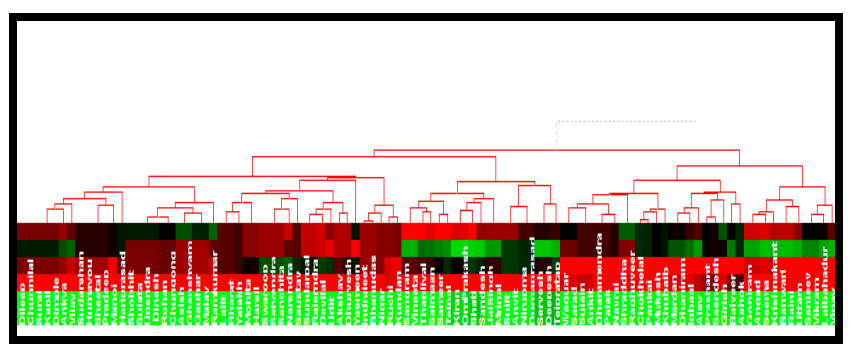

Figure 3: Dendrogram of Shor Algorithm based Cancer Disease Detection Technique using Euclidean Distance.

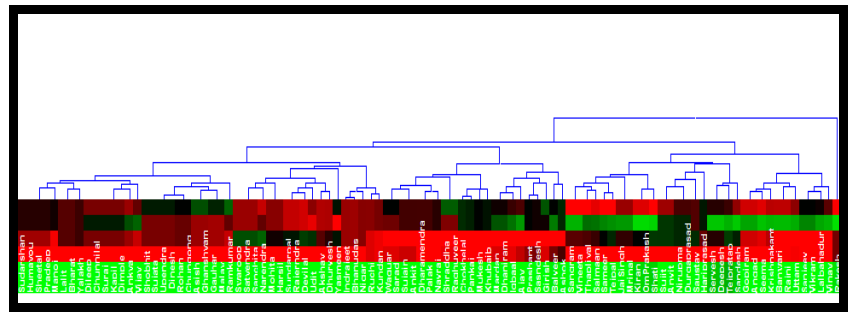

Figure 4: Dendrogram of Shor Algorithm based Cancer Disease Detection Technique using Euclidean Distance.

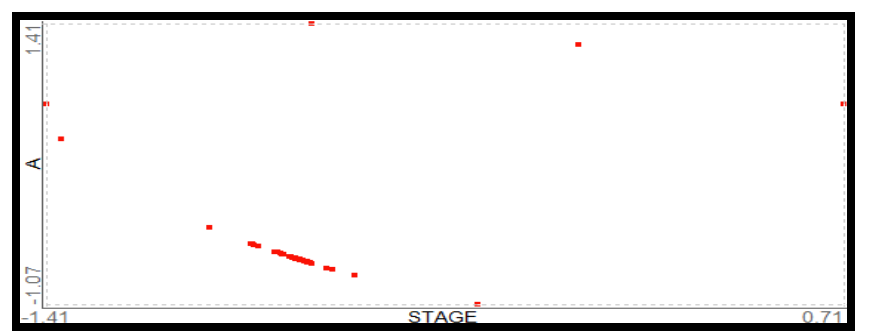

Figure 5: Scatter plot Ordering of Conventional Cancer Disease Detection Technique using Euclidean Distance.

Stages, tumor size, Random number, Node status and Metastasis. The Figure 3 is based on Euclidean Distance shows Number used, Stages, tumor size, Random number, Node status and Metastasis condition from upper layer to lower most layers consecutively.

For K-means clustering technique: The Result of K-means clustering technique for Shor Algorithm based Cancer disease detection System these Dendrogram are shows Stages, tumor size, Random number, Node status and Metastasis.

The Figure 4 is based on Euclidean Distance shows Number used, Stages, tumor size, Random number, Node status and Metastasis condition from upper layer to lower most layers consecutively.The major benefit of using these parameters in analyses is reduction in error. The color intensities and changes in color are same as in above Dendrogram. These results are seen with red color and high in intensity on Stage showing layer i.e. $2^{\text {th }}$ layer in $1^{\text {st }}$ and $3^{\text {rd }}$ Dendrogram and as lowermost layer in $2^{\text {nd }}$ Dendrogram. In comparison to Conventional Technique here the stages also showing effect of Node status and metastasis which can be confirmed form Table 1 and 2 easily. With the help of this information Shor Algorithm based Cancer Detection Technique becomes more efficient than Conventional Technique.

Results comparison between conventional cancer disease detection technique and shor algorithm based cancer disease detection technique on the basis of scatter plot ordering

In Scatter plot ordering the data is displayed as a collection of points, each having the value of one variable determining the position on the horizontal axis and the value of the other variable determining the position on the vertical axis. Here the Scatter plot is plotted between Tumor size (A) and stages of Cancer. Afterclustering of result using different Distance measures/Similarieies the Scatter plot ordering obtained is same in all the cases as given in the following figures. This discussion is also devided into two sections in which Dendrogram of conventional cancer disease detection technique contains the results of Conventional technique where as Dendrogram of Shor algorithm based cancer disease detection technique contains the results of Shor Algorithm based results. 
Scatter plot ordering of conventional cancer disease detection technique: In this section the Results of Conventional Cancer Disease Detection System in the form of Scatter plots are illustrated.

The Figure 5 contain the Stages of cancer on the $\mathrm{X}$-axis whereas Tumor size (A) on the $\mathrm{Y}$-axis. The points shown in red color represents the stages on aparticular tumor size.In the stage axis the points -1.41 to 0.71 shows the minimum and maximum values and all the records lies between these points and in the same respect illustrated in the tumor size axis i.e. the data lies between -1.07 to 1.41. The Scatter plot shows the stages of cancer according to tumor size only.

Scatter plot ordering of shor algorithm based cancer disease detection technique

For agglomerative hierarchical clustering technique: The Result of Agglomerative Hierarchical clustering technique for Shor Algorithm based Cancer disease detection System is shown here. Y-axis is dedicated for Stages and X-axis for Tumor size (A). In the Figure 6 the results which are shown are based on Agglomerative Hierarchical Clustering using Euclidean distance.

For K-means clustering technique: The Figure 7 is the result of Shor Algorithm based Cancer Disease Detection Technique using Euclidean Distance. In the A (Tumor size) axis the points -0.39 to 1.98 shows the minimum and maximum value and all the records lies between these points. In the same way on the Stage axis i.e. the data lies between -0.89 to 1.06 . The Scatter plot ordering show the changes occur in stages of cancer according to Cancer tumor size. This Scatter plot ordering is able to give a more clear idea about the patient condition as when we see the differences in stages not only due to tumor size but also due to metastasis condition and node status. After observing Scatter plot ordering of both the Techniques i.e. conventional one and Quantum Computing based it is clearly visible that efficiency in term of data analysis is more in $2^{\text {nd }}$ one.

Results comparison between conventional cancer disease detection technique and shor algorithm based cancer disease detection technique on the basis of graphical representations

This is another way to get a good comparative analyses of results

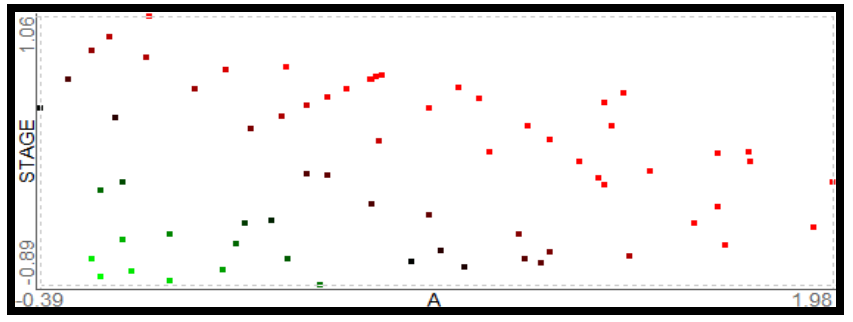

Figure 6: Scatter plot Ordering of Shor Algorithm based Cancer Disease Detection Technique using Euclidean Distance.

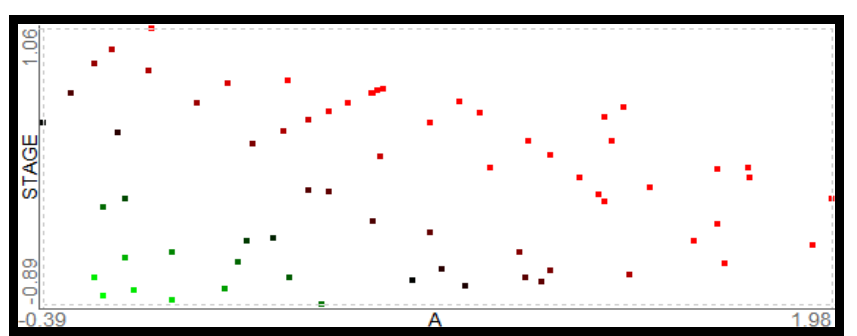

Figure 7: Scatter plot Ordering of Shor Algorithm based Cancer Disease Detection Technique using Euclidean Distance.

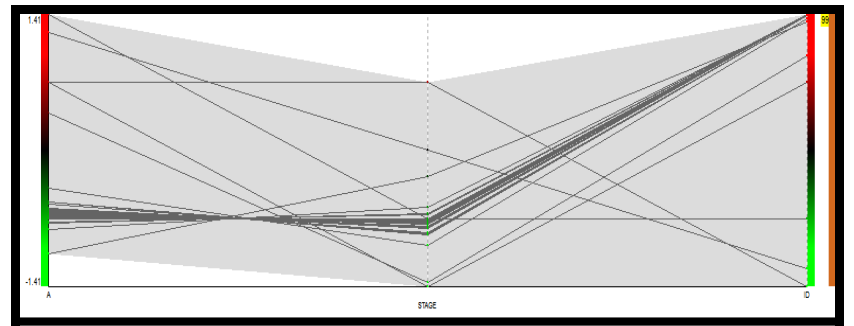

Figure 8: Graphical Representation of Conventional Cancer Disease Detection Technique using Euclidean Distance.

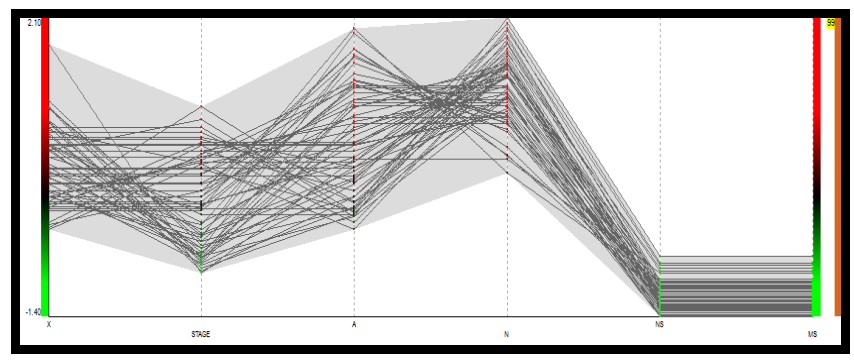

Figure 9: Graphical Representation of Shor Algorithm based Cancer Disease Detection Technique using Euclidean Distance.

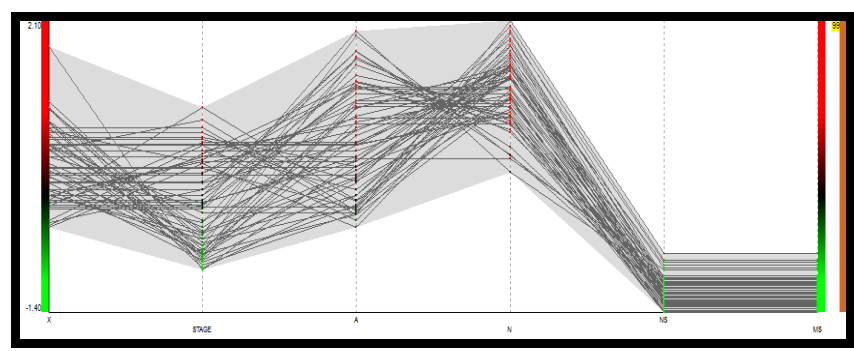

Figure 10: Graphical Representation of Shor Algorithm based Cancer Disease Detection Technique using Euclidean Distance.

as the following Figures shows no differences it means the clustering by any of the Distance measures do not affect the results adversly. This part is also devided into two sections.

Graphical representations of conventional cancer disease detection technique: The Figure 8 shows the results of Coventional Cancer Disease Detection Technique using Euclidean Distance. On the stage axis in this profile search the above most point shows the patient having most severe Disease whereas the lower most point shows the patient which is least affected

Graphical representation of shor algorithm based cancer disease detection technique

For Agglomerative hierarchical clustering technique: The Figure 9 illustrate the Results of Shor Algorithm based Cancer Disease Detection Technique using Euclidean Distance as Distance measure when doing Agglomerative Hierarchical Clustering.

For K-means clustering technique: The Figure 10 illustrate the Results of Shor Algorithm based Cancer Disease Detection Technique using Euclidean Distance as Pearson Correlation Coefficientwhen doing K-means Hierarchical Clustering.

These graphs show the report of the Cancer Patients. In these 
Graphs Tumor size (A), Metastasis Condition (MS), Node Status (NS) helps us to detect the exact Stage of Cancer. After observing these results which use different Distance Measures/Similarities the result perception do not change which indicate the efficiency of the proposed technique.

By these two profile search it is easy to understand that there is Shor Algorithm which is a basic Algorithm of Quantum Computing is giving results convenient for an Oncologist and make an exact figure of a patient's condition who suffers from the most devastating disease of this century i.e. Cancer (Cancer is a leading cause of death worldwide, accounting for 7.6 million deaths i.e. around $13 \%$ of all deaths in 2008) as here in this proposed work the use of tumour size as a parameter for disease prediction with metastasis condition and node status is used so accuracy is also enhanced in compare to any technique which uses only tumour size as a parameter. So the chances of error in disease detection increases because in many cases this is observed that a patient having a large tumour size have not been suffering from cancer, as the node status is not shows symptoms of Cancer (N0) also the possibility of Metastasis condition where the tumour is not showing any malignancy (M0) but in case of Quantum Computing based Approach i.e. Shor Algorithm based Cancer Disease Detection Technique these errors has been removed for increasing accuracy.

The different Hierarchical Clustering helps to understand the results by making clusters of acquired data obtained by both the Detection Techniques.

Finally with the help of these three Distance measures we get difference among the conventional approach and Quantum Computing based approach. Where Conventional Approach which is used widely for Cancer Disease Detection-based on Classical Computing, on the other hand this Shor Algorithm based Cancer Disease Detection technique is totally based on Quantum Computing so an Oncologist get the results more frequently that is within a few seconds in compare to hours in case of Classical Computing.

Also the result are more accurate and accessibility to different parameters is more in case of Quantum Computing based approach so it is much easy for an oncologist to create a treatment program with more ease and accuracy within a short period of time which may be act as a boon for the Cancer patients in near future.

NOTE- This procedure is not applicable for Leukemia and brain tumour.

\section{Conclusion}

A quantum computing based technique start work after diagnoses of cancer, and by quantum computing based Algorithms getting exact analyses is easy, here with the help of this an Oncologist can increase the treatment efficiency. It provides an interrelation and makes a more appropriate approach for accurate disease detection. In collaboration with various Hierarchical Clustering Techniques, the Shor Algorithm based Cancer Disease Detection Technique gives a different analytical approach which helps a lot to understand the results provided by our proposed system.

\section{Future Scopes}

Quantum Computing have a great opportunity in the field of Medicine where the various Disease affects the mankind \& other Organisms. Different parameter of Cancers further can be used for betterment of the Diagnosis Scenario of Cancer, where as far other Disease may also get benefits from the different other Algorithm of
Quantum Computing in future. In near future as the healthcare sector need a tremendous need of techniques and have an opportunity of great economic growth so, this type of work can be utilize to fetch attention for various aspects.

\section{References}

1. Vishal Sahni (2010) Quantum Computing. Tata McGraw Hill, New Delhi.

2. David Deutsch (1992) Quantum computation. Physics World.

3. Cue the qubits: Quantum computing - How to make a quantum computer. The Economist 342: 79-81.

4. Two-bit heroes -Computing with quanta. The Economist 338: 7948

5. Lloyd S (1996) Universal Quantum Simulators Science 273: 1073-1078.

6. Oeztuerk-Winder F, Ventura JJ (2012) The many faces of p38 mitogen-activated protein kinase in progenitor/stem cell differentiation. Biochem J 445: 1-10.

7. Anand $P$, Kunnumakkara AB, Sundaram C, Harikumar KB, Tharakan ST, et al. (2008) Cancer is a preventable disease that requires major lifestyle changes. Pharm Res 25: 2097-2116.

8. Kinzler, Kenneth W, Vogelstein, Bert (2002) The genetic basis of human cancer New York: McGraw-Hill.

9. Nahleh ZA (2006) Hormonal therapy for male breast cancer: A different approach for a different disease. Cancer Treat Rev 32: 101-105.

10. Childs AM, Schulman LJ, Vazirani, Umesh V (2007) Quantum Algorithms for Hidden Nonlinear Structures. Foundations of Computer Science, 48th Annual IEEE Symposium 395-404

11. Fortunato Bianconi, Valerio Brunori, Paolo Valigi, Francesco La Rosa, Fabrizio Stracci (2012) Information Technology as Tools for Cancer Registry and Regional Cancer Network Integration. IEEE Transaction on Systems, Man and Cybernetics-part A: Systems and Humans 42: 1410 - 1424.

12. Guilu Long, Tzyh Jong Tarn (2008) Progress in theoretical quantum computing Front. Computer Sci. China, 2: 113.

13. Wong HS, Wang HQ (2008) Constructing the gene regulation-leve representation of microarray data for cancer classification. J Biomed Inform 41: 95-105.

14. Hualong Yu, Jun Ni, Yuanyuan Dan, Sen Xu (2012) Mining and Integrating Reliable Decision Rules for Imbalanced Cancer Gene Expression Data Sets. Tsinghua Science and technology 17: 666-673.

15. Seker H, Odetayo MO, Petrovic D, Naguib RN (2003) A fuzzy logic basedmethod for prognostic decision making in breast and prostate cancers. IEEE Trans Inf Technol Biomed 7: 114-122.

16. Jiang Min, Zhang Zengke, Tzyh-Jong Tarn (2008) Efficient Scheme for Optimizing Quantum Fourier Circuits. Tsinghua Science and Technology 13: 54-58.

17. Jiang Min, Huang Xu, Zhou Yiming (2011) Quantum Switching Based on the Nearest Neighbour Hamiltonian. Tsinghua Science and Technology 16: 100-105.

18. Garibaldi JM, Zhou SM, Wang XY, John RI, Ellis IO (2012) Incorporation of expert variability into breast cancer treatment recommendation in designing clinical protocol guided fuzzy rule system models. J Biomed Inform 45: 447459.

19. Kondo T, Ueno J, Takao S (2012) Hybrid Multi-layered GMDH-Type Neura Network Using Principal Component-Regression Analysis and Its Application to Medical Image Diagnosis of Lung Cancer. Bio-Medical Computing 20- 27.

20. Leila HE, Caroline BR, Anthony JF, Vincent PW (2013) Optimizing multidimensional terahertz imaging analysis for colon cancer diagnosis. Expert Systems with Applications 40: 2043-2050.

21. Wang L, Chu F, Xie W (2007) Accurate cancer classification using expressions of very few genes. IEEE/ACM Trans Comput Biol Bioinform 4: 40-53.

22. Lov KG (1999) How fast can a quantum computer search. Quant-ph 1-7.

23. Lov KG (2001) From Schrödinger's Equation to the Quantum Search Algorithm. quant-ph.

24. Shi Kuifan, Chen Yuehui, Dong Jiwen (1997) Classification of Cancer Cells 
Citation: Jain M, Chaturvedi SK (2014) Quantum Computing Based Technique for Cancer Disease Detection System. J Comput Sci Syst Biol 7: 095102. doi: $10.4172 / j c s b .1000143$

Using Neural Networks in Combination with Expert Experience. Tsinghua Science and Technology 2: 853-855.

25. Walker HL, Xinpei Ma, Erin Barnes, Xingye Qiao, John Heine, at al. (2012)
PNN/GRNN Ensemble Processor Design for Early Screening of Breast Cancer Procedia Computer Science 12: 438-443.

26. Yuichiro Fujiwara, Vladimir DT (2013) A Characterization of EntanglementAssisted Quantum Low-Density Parity-Check Codes. IEEE transactions on information theory 59: 3347-3353. 